\title{
The sulcus line of the trochlear groove is more accurate than Whiteside's Line in determining femoral component rotation
}

\author{
Simon Talbot $\cdot$ Pandelis Dimitriou $\cdot$ \\ Ross Radic $\cdot$ Rachel Zordan $\cdot$ John Bartlett
}

Received: 15 January 2014/ Accepted: 5 June 2014/Published online: 1 July 2014

(C) Springer-Verlag Berlin Heidelberg 2014

\begin{abstract}
Purpose The sulcus line (SL) is a three-dimensional curve produced from multiple points along the trochlear groove. Whiteside's Line, also known as the anteroposterior axis (APA), is derived from single anterior and posterior points. The purposes of the two studies presented in this paper are to (1) assess the results from the clinical use of the SL in a large clinical series, (2) measure the SL and the APA on three-dimensional CT reconstructions, (3) demonstrate the effect of parallax error on the use of the APA and (4) determine the accuracy of an axis derived by combining the SL and the posterior condylar axis (PCA). Methods In the first study, we assessed the SL using a large, single surgeon series of consecutive patients undergoing primary total knee arthroplasties. The post-operative CT scans of patients $(n=200)$ were examined to determine the final rotational alignment of the femoral component. In the second study, measurements were taken in a series of 3DCT reconstructions of osteoarthritic knees $(n=44)$.
\end{abstract}

S. Talbot $(\bowtie) \cdot$ P. Dimitriou $\cdot$ R. Radic

Western Health, Melbourne, VIC, Australia

e-mail: info@simontalbot.com.au

P. Dimitriou

e-mail: pdimitriou@me.com

R. Radic

e-mail: rossradic@gmail.com

S. Talbot $\cdot$ R. Zordan $\cdot$ J. Bartlett

Warringal Private Hospital, Melbourne, VIC, Australia

e-mail: rachelzordan@gmail.com

J. Bartlett

e-mail: r.j.bartlett@bigpond.com.au
Results The mean position of the femoral component in the clinical series was $0.6^{\circ}$ externally rotated to the surgical epicondylar axis, with a standard deviation of $2.9^{\circ}$ (ranges from $-7.2^{\circ}$ to $6.7^{\circ}$ ). On the $3 \mathrm{DCT}$ reconstructions, the APA $\left(88.2^{\circ} \pm 4.2^{\circ}\right)$ had significantly higher variance than the $\mathrm{SL}\left(90.3^{\circ} \pm 2.7^{\circ}\right)(F=5.82$ and $p=0.017)$. An axis derived by averaging the SL and the PCA $+3^{\circ}$ produced a significant decrease in both the number of outliers ( $p=0.03$ vs. PCA and $p=0.007$ vs. SL) and the variance $(F=6.15$ and $p=0.015$ vs. SL). The coronal alignment of the SL varied widely relative to the mechanical axis $\left(0.4^{\circ} \pm 3.8^{\circ}\right)$ and the distal condylar surface $\left(2.6^{\circ} \pm 4.3^{\circ}\right)$. Conclusions The multiple points used to determine the SL confer anatomical and geometrical advantages, and therefore, it should be considered a separate rotational landmark to the APA. These findings may explain the high degree of variability in the measurement of the APA which is documented in the literature. Combining a geometrically correct SL and the PCA is likely to further improve accuracy.

Keywords Knee · Arthroplasty · Rotation - Femoral component rotation - Total knee arthroplasty · Whiteside's Line $\cdot$ Sulcus line $\cdot$ Epicondylar axis

\section{Introduction}

Successful total knee arthroplasty (TKA) is dependent on the accurate alignment of the components. Inaccurate femoral component rotation is associated with poor outcomes due to patellofemoral maltracking, flexion instability, and soft tissue imbalance [1, 2, 5, 7, 15, 34]. Obtaining correct femoral component alignment is difficult as the definitive landmarks are not readily accessible during 
surgery, and therefore surrogate landmarks and techniques are adopted [21-23, 37].

Several techniques are commonly used to determine the rotational alignment of the femoral component. Measured resection techniques use surface-derived landmarks to direct the rotational alignment of the implant. The most frequently referenced bony landmarks are the posterior condylar axis (PCA) [16, 19, 24], the surgical epicondylar axis (SEA) [4, 17, 42], the anatomical epicondylar axis (AEA) [33, 46], and the anteroposterior axis (APA) [3, 27, 43]. The gap-balancing technique [13] attempts to achieve a balanced knee by beginning with the tibial resection perpendicular to the tibial axis, and subsequent femoral resection based on ligament tensioning from this cut surface. The use of these techniques has continued to produce high rates of unacceptable femoral component malrotation $[25,36,38,40]$. As a result, alternative techniques employing preoperative CT scans, computer navigation, patient-specific instrumentation (PSI), or the combination of landmarks are being considered [38, 40].

Based on anatomical and kinematic data, the accepted gold standard for correct femoral component rotation is the SEA measured on a CT scan $[2,4,11,28,30]$. Unfortunately, both epicondyles are relatively broad structures covered in dense soft tissue in vivo making them difficult to identify intraoperatively [3, 22, 39]. Many studies comparing landmarks are difficult to interpret as they fail to use post-operative CT scans referencing the SEA [14, 24].

The sulcus line (SL) is a curve derived from joining multiple points in the trochlear groove. In practice, many surgeons use a version of the SL by marking out a line along the depth of the trochlear groove. In comparison, the APA, also known as Whiteside's Line, is defined as the axis taken from two points-the deepest part of the patella groove anteriorly to the centre of the intercondylar notch posteriorly [3]. Both anatomical and clinical studies suggest that the APA is an inaccurate axis and that it is difficult to consistently reproduce [27, 33, 37, 40, 44].

It is hypothesised that there are two reasons why the SL is more accurate than the APA. Firstly, the APA relies on the accuracy of the anterior point which is in the proximal section of the trochlear. This section is often affected by osteoarthritis and dysplasia. The SL references the vertical section extending anteriorly from the intercondylar notch into the anterior trochlear groove, but does not rely on the proximal section of the trochlear. Secondly, even though both axes reference the trochlear groove, the SL has geometrical advantages which make it a more accurate landmark. The rotational component of the trochlear groove can be isolated by viewing the SL along the coronal axis of the trochlear groove. This axis can be identified intraoperatively, and on three-dimensional CT reconstructions, as the viewpoint at which the curved SL becomes a straight, vertical line. Conversely, as the APA is only derived from two points, there is no way in which the rotational component of the trochlear groove can be isolated.

The following investigation comprises of two studies. The first study is a retrospective review of post-operative CT scans in 200 total knee replacements in which we aim to (1) determine the femoral component rotational alignment achieved with the use of the SL and (2) compare these results with other studies which have used post-operative CT scans to assess rotational axes. The second study examines the anatomical landmarks in preoperative threedimensional CT reconstructions of 44 osteoarthritic knees. It aims to (1) compare a geometrically correct SL measured along the coronal axis of the trochlear groove to other anatomical landmarks, including the APA, (2) demonstrate the effect of parallax error on the measurement of the APA, and (3) measure the accuracy and variability of an axis derived by combining the PCA and a geometrically correct SL.

\section{Materials and methods}

\section{Study 1}

A retrospective review of a consecutive case series of patients undergoing primary TKA by one surgeon from July 2008 to June 2009 was conducted to document the femoral rotation of the prosthesis on post-operative CT scans $(n=228)$. Excluded from the study were CT scans which were determined by either of the assessors as not providing adequate visualisation of the landmarks due to metallic artefact $(n=28)$, leaving 200 cases.

\section{Surgical technique}

All TKAs were performed by one surgeon, very experienced in TKA, and who has used the SL extensively for many years. The same surgical approach, consisting of a midline skin incision and a medial parapatellar approach, was applied to all patients. Conventional instruments were used, with intramedullary (IM) femoral alignment and extramedullary tibial alignment jigs. Prostheses inserted were the Triathlon (Stryker Howmedica Osteonics, Allendale, NJ) or Active (Advanced Surgical Design and Manufacture, ASDM, Sydney, Australia) knee. All components were cruciate retaining, and all femoral components were uncemented.

The femoral rotational alignment was based only on the SL. The line was meticulously drawn by palpating the trochlear groove. Multiple diathermy marks were made in the deepest part of the groove at the tip of the thumb. The marks were extended from the anterior edge of the 
intercondylar notch to the proximal trochlear groove. The most proximal aspect of the trochlear groove was disregarded if it was found to deviate from the rest of the groove.

The SL was then viewed along the coronal plane orientation that produced a straight, vertical line (Fig. 1). When the SL is viewed along a different coronal orientation, it becomes a curve (Fig. 2). This vertical line was then transferred onto the surface of the distal condyles with a perpendicular T-piece. The horizontal limb was marked with a further diathermy line (Fig. 3).

An IM rod was inserted through the centre of the knee. The anterior femoral cut was made first with a cutting block attached to the IM rod which was rotated to match the horizontal diathermy line. A stylus was used to avoid notching. The distal cut was made by pinning a flat distal cutting block onto the anterior surface. The appropriately sized 4-in-1 cutting block was then placed on the distal cut

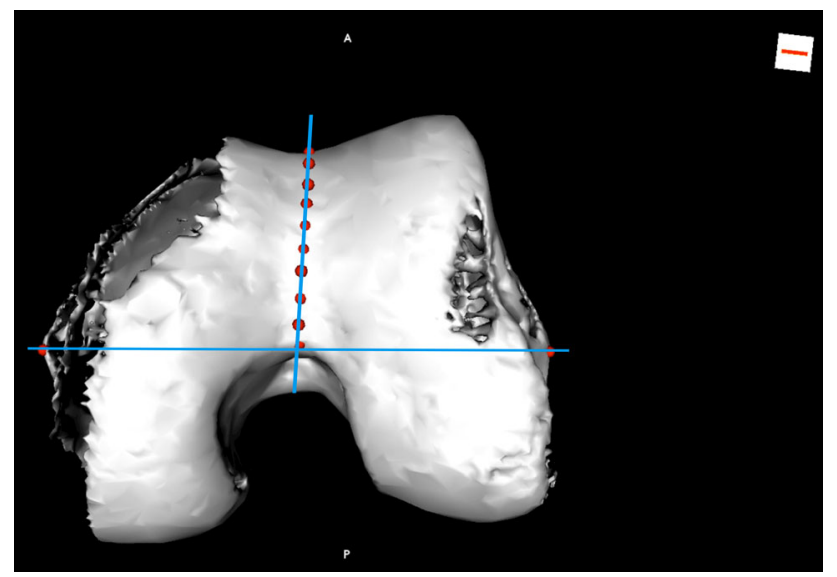

Fig. 1 The SL is a straight line when it is viewed along the coronal alignment of the trochlear groove

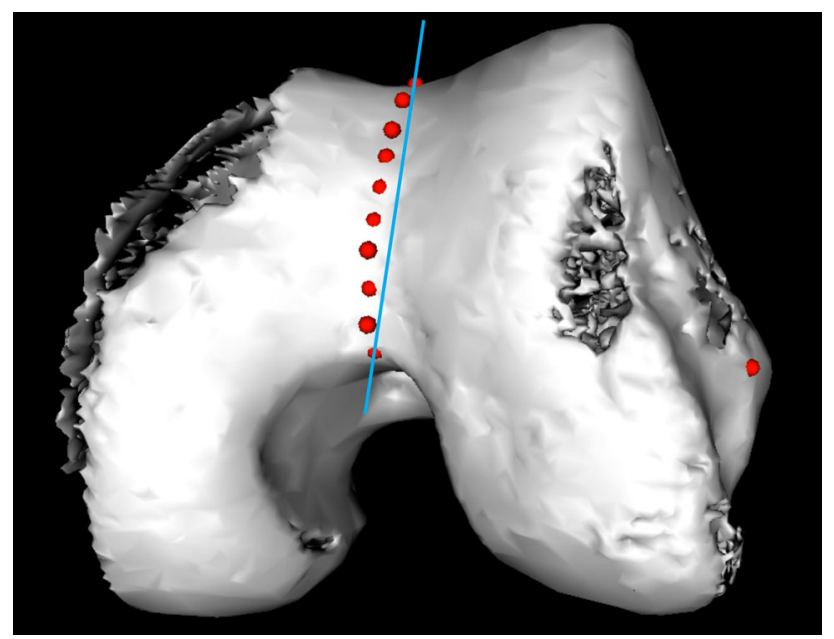

Fig. 2 The SL appears as a curve when it is not viewed along the coronal alignment of the trochlear groove

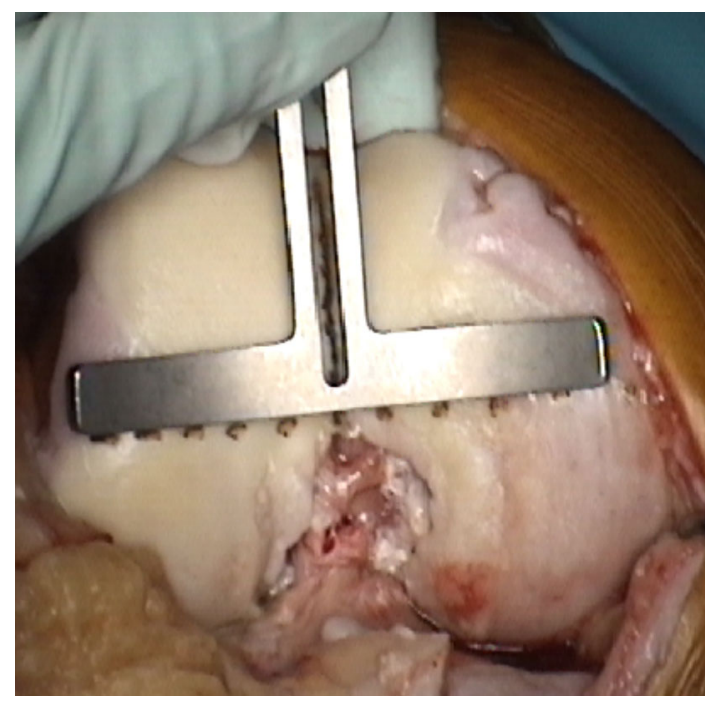

Fig. 3 A T-piece was used to translate the vertical SL into a horizontal line

surface with an anterior ledge which sat on the anterior femoral cut. This was pinned, and the posterior and chamfer cuts were completed.

\section{Post-operative CT evaluation}

An initial scanogram was produced, and $1.25-\mathrm{mm}$ slices were performed from approximately $5 \mathrm{~cm}$ above the anterior flange of the femoral component to immediately below the tibial stem.

The medial and lateral epicondyles were identified. The AEA was measured from the most prominent point on the lateral epicondyle to the most prominent point on the medial epicondyle. The medial sulcus was identified where possible. If the sulcus was not visible, the absence of a sulcus was documented. The SEA was measured from the most prominent point on the lateral epicondyle to the centre of the sulcus of the medial epicondyle as outlined by Berger [8]. The rotational alignment of the femoral component was measured from the under surface of the anterior flange, in the manner described by Moon et al. [26], as this surface was less affected by the artefact than the posterior condyles. All measurements were taken and reported to one decimal place.

Two assessors independently reviewed the CT scans and calculated the measurements. Intraobserver and interobserver reliability was both very good (intraobserver $r=0.95$ and 0.97 and interobserver $r=0.93$ ).

\section{Statistical analysis}

Analyses to determine intraclass correlation coefficients (ICC), means, standard deviation (SD), and ranges were 


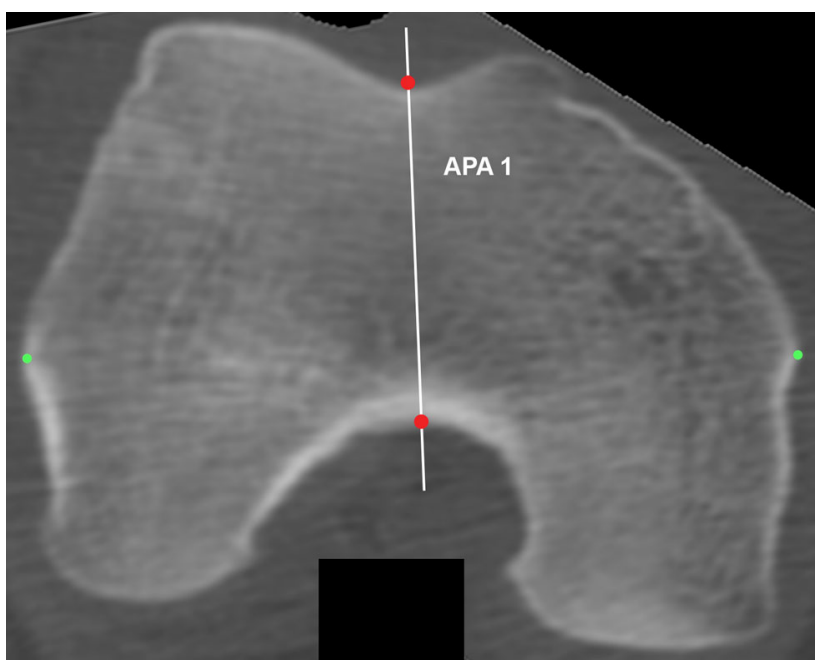

Fig. 4 APA-1 was measured on a single CT slice

conducted. Pearson's correlation and independent samples $t$ tests were conducted to ascertain the relationships between age, gender, and side with the rotation of the femoral component. All analyses used the Statistical Package for the Social Sciences (SPSS) v.16.0.

\section{Study 2}

This was a retrospective review of a consecutive series of preoperative CT scans of patients who were undergoing TKA for severe osteoarthritis.

In total, $44 \mathrm{CT}$ scans were assessed. All scans were performed using the Perth protocol [10], and 1.25-mm slices were performed from hip to ankle (GE Optima 660 Brightspeed, 128 slice scanner). The data were imported into the Osirix (Osirix v.5.6 64-bit, Pixmeo Sarl, Switzerland) proprietary software program.

A volume rendering three-dimensional (3D) reconstruction was performed. Bone subtraction techniques were used to remove the patella and tibia. A 3D surface rendering was then performed. The medial and lateral epicondyles and the medial epicondylar sulcus were viewed in multiple planes and marked with region of interest (ROI) points. These points were then used as the reference points for both the two-dimensional and three-dimensional measurements.

The first measurements were taken from two-dimensional axial slices in order to recreate the techniques used in previous studies. The first technique for measuring the APA (APA-1) was determined by marking the deepest point in the groove anteriorly and the midpoint in the notch posteriorly as per the technique used by Nagamine et al. [27] (Figs. 4, 5). Measurements were taken on the axial slice that most clearly showed the epicondyles and medial epicondylar sulcus. The AEA, SEA, and PCA were measured as per the technique described by Berger et al. [8].

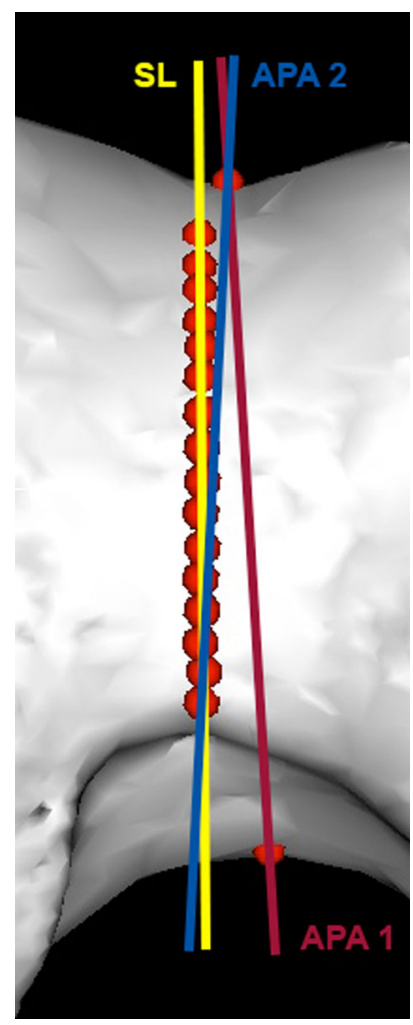

Fig. 5 The SL is compared to the two-dimensional (APA-1) and three-dimensional (APA-2) techniques for determining the APA

The deepest points in the trochlear groove were marked as ROIs on the surface rendering and confirmed using the orthogonal views in the MPR (multi-planar reconstruction) format as per the technique used by Iranpour et al. [20]. Following confirmation of 8-12 ROI points, the groove was viewed as a $3 \mathrm{D}$ surface rendering which was rotated along the axis of the SL in order further assess the accuracy of the points (Figs. 6, 7).

The APA and SL were then measured with the femur orientated along the mechanical axis (MAx). The 3D surface rendering was rotated in each plane until the femoral head was in line with the centre of the knee. The angles were measured relative to the ROIs placed on the epicondyles (SEA). The second technique for measuring the APA (APA-2) was measured from the deepest point on the notch anteriorly to the closest point to the intercondylar notch on the articular surface posteriorly as per technique described by Victor et al. [42] (Fig. 5). The SL was also measured on the same image. When the SL was noted to be curved, due to variation between the coronal alignment of the SL and the MAx, a best fit was measured concentrating on the vertical section of the line.

The 3D surface rendering was then aligned along the coronal axis of the SL (CAxSL) by rotating the coronal alignment of the reconstruction until the straightest 

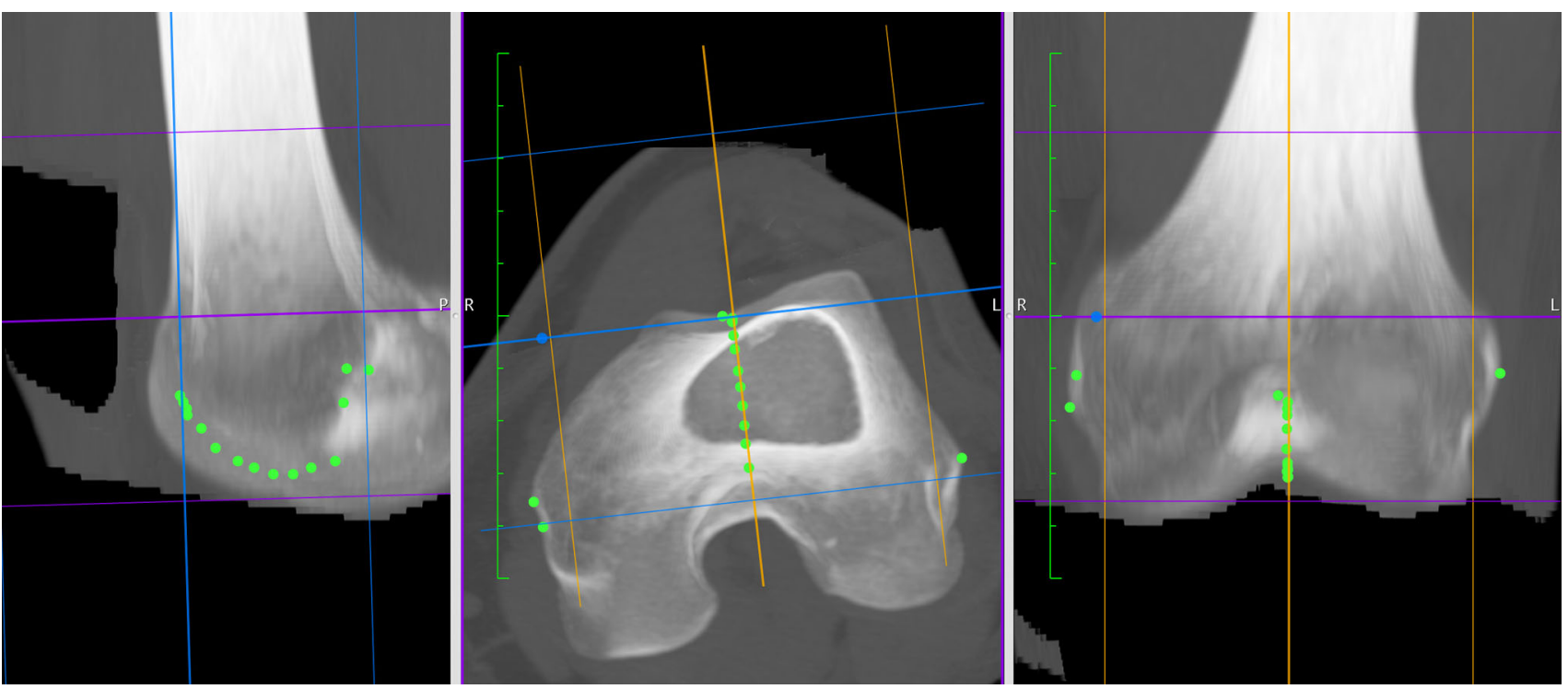

Fig. 6 The trochlear groove and epicondyles were marked on multi-planar reconstructions

possible version of the SL was visible. This technique most closely matches the intraoperative technique of altering the viewpoint of the surgeon until the SL appears to be a straight line rather than the shallow curve that is apparent when it is viewed in an oblique plane (Figs. 1, 2).

The CAxSL was then measured on the MPR screen in comparison with the MAx (Fig. 6). The femur was orientated to the MAx in the sagittal plane and to the SL in the axial plane. The SL was then viewed as a straight line in the coronal plane. A line was extended along the CAxSL towards the hip and was measured relative to the MAx. A varus deviation (towards the anatomical axis) was assigned a positive value, and valgus deviation was assigned a negative value. The distal condylar axis (DCA) was measured as the angle between the MAx and a line across the distal surface of the condyles.

The ROI points were inserted by a single observer. To assess intraobserver reliability, insertion of the points was repeated for a second time in ten femurs at least a week later. The intraobserver reliability was found to be very good $(r=0.93)$. All measurements were taken by two assessors (an orthopaedic surgeon and a registrar). Interobserver reliability was also found to be very good, with $r=0.93$.

\section{Statistical analysis}

Analyses to determine ICC, means, SD, and ranges were conducted. $T$ tests and one-way ANOVA were applied to compare groups. Outliers, beyond $3{ }^{\circ}$ from the SEA, were determined for each group and comparative analysis between each groups conducted using chi-square test.

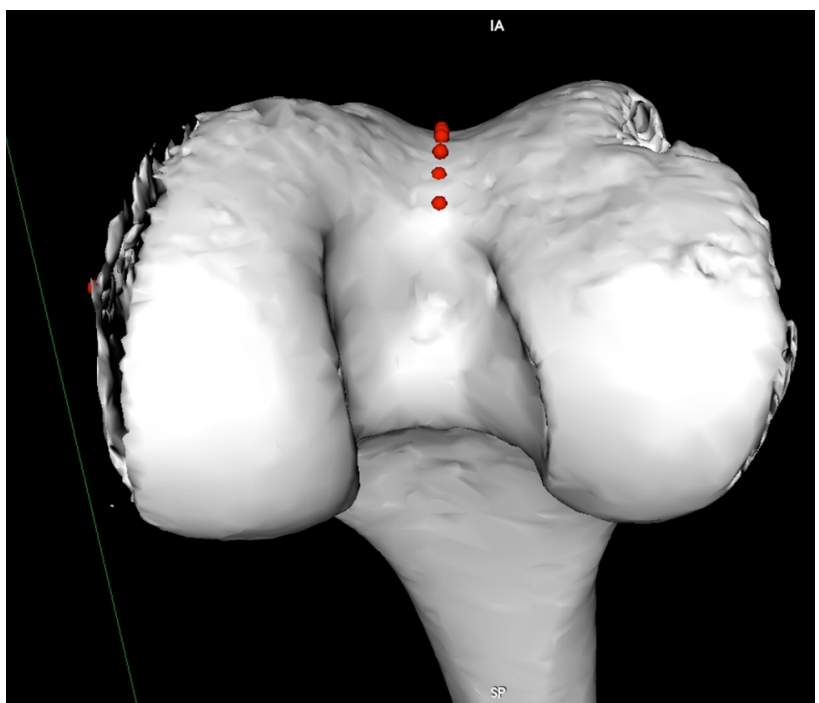

Fig. 7 The landmarks were confirmed by rotating 3-D reconstructions

Significance was set at the $p \leq 0.05$ level. SPSS v.16.0 was used for all analyses.

Ethics approval was sought and received for each study from the Western Centre for Health Research Education (QA2013.45 and QA2013.46).

\section{Results}

Study 1

The average age of participants was 68 years $(\mathrm{SD}=8.9)$. See Table 1 for demographic data. 
The sulcus was identified in 181 (91\%) scans. Thus, the AEA and the SEA were calculated on 200 and 181 scans, respectively. The results of this are summarised in Table 2 .

Of the 181 knees with an identified sulcus, 64 (35\%) were deemed outliers, with deviation greater than $3^{\circ}$ from the SEA. Of these outliers, 16 deviated greater than $5^{\circ}$ from the SEA.

Male patients had a significantly externally rotated femoral component position $\left(\right.$ mean $\left.=1.04^{\circ}\right)$ compared with female patients $\left(\right.$ mean $=0.17^{\circ}, t=2.04$, and $p=0.043$ ). All remaining analyses were non-significant.

\section{Study 2}

There was a significant difference between the SEA and the APA-1 and APA-2 $(p<0.05)$. There was no significant difference between the SEA and the SL measured along the MAx, the SL measured along the CAxSL, the PCA $+3^{\circ}$, or the average of PCA+3 and SL. The axial rotational measurements and results of the comparative analysis are outlined in Table 3 and summarised in Fig. 8.

The variability of the SL was significantly less than the variability of the APA-2 on one-way ANOVA $(F=5.82$ and $p=0.017)$. The axis derived from the average of the $\mathrm{PCA}+3$ and the SL was significantly less variable than the $\mathrm{SL}(F=6.15$ and $p=0.015)$. The SL on 3D reconstruction measured along the MAx had a higher SD than the SL measured along the CAxSL $\left(3.2^{\circ}\right.$ vs. $2.7^{\circ}$ ); however, the difference in variance did not reach statistical significance $(F=3.71, p=0.052)$.

The number of outliers, greater than $3^{\circ}$ from the SEA, was calculated for the PCA $+3^{\circ}$ and the SL along the

Table 1 Demographic data

\begin{tabular}{lrr}
\hline Variable & $n$ & $\%$ \\
\hline Gender & & \\
Male & 101 & 51 \\
Female & 99 & 49 \\
Side & & 48 \\
Left & 95 & 52 \\
Right & 105 & \\
Prosthesis & & 59 \\
Active & 117 & 41 \\
Triathlon & 83 & \\
\hline
\end{tabular}

Table 2 Axial alignment of femoral components relative to epicondylar axes

\begin{tabular}{lrlr}
\hline & Mean & SD & \multicolumn{1}{l}{ Range } \\
\hline Component to AEA & $-3.2^{\circ}$ & $2.9^{\circ}$ & $-10.8^{\circ}$ to $3.2^{\circ}$ \\
Component to SEA & $0.6^{\circ}$ & $2.9^{\circ}$ & $-7.2^{\circ}$ to $6.7^{\circ}$ \\
\hline
\end{tabular}

CAxSL. A combined axis was calculated by averaging the $\mathrm{PCA}+3^{\circ}$ and the SL along the CAxSL for each knee (PCA-SL). This derived axis produced a mean of $0.5^{\circ}$ (SD $2.2^{\circ}$, range from $-4.8^{\circ}$ to $5.2^{\circ}$ ). The PCA-SL was found have significantly less outliers when compared with either the $\mathrm{PCA}+3^{\circ}$ or the SL along CAxSL individually, with $p$ values of 0.03 and 0.007 , respectively. For all outlier data, see Table 4.

The coronal axis measurements are summarised in Table 5.

\section{Discussion}

There are several important findings from these studies. Firstly, the SL should be considered a separate, and more accurate, rotational landmark than the APA. Secondly, the

Table 3 Rotational variations between axes and variations in coronal viewpoint

\begin{tabular}{lrrll}
\hline & Mean & SD & Range & $\begin{array}{l}\text { Difference } \\
\text { from SEA }\end{array}$ \\
\hline AEA to SEA & $3.7^{\circ}$ & $0.6^{\circ}$ & $2.4^{\circ}-4.8^{\circ}$ & N/A \\
APA-1 on 2D axial & $-1.5^{\circ}$ & $3.6^{\circ}$ & $5^{\circ}$ to $-8.2^{\circ}$ & $p=0.008$ \\
PCA+3 & $0.7^{\circ}$ & $2.5^{\circ}$ & $7.1^{\circ}$ to $-5.7^{\circ}$ & n.s. \\
APA-2 on 3D & $-1.8^{\circ}$ & $4.2^{\circ}$ & $7.8^{\circ}$ to $-11.8^{\circ}$ & $p=0.005$ \\
$\quad$ reconstruction & & & & \\
SL along MAx & $0.0^{\circ}$ & $3.2^{\circ}$ & $5.1^{\circ}$ to $-7.4^{\circ}$ & n.s. \\
SL along CAxSL & $0.3^{\circ}$ & $2.7^{\circ}$ & $4.7^{\circ}$ to $-4.9^{\circ}$ & n.s. \\
Mean PCA+3 ${ }^{\circ}$ and & $0.5^{\circ}$ & $2.2^{\circ}$ & $5.2^{\circ}$ to $-4.8^{\circ}$ & n.s. \\
$\quad$ SL along CAxSL & & & & \\
\hline
\end{tabular}

Results are all relative to the SEA. $90^{\circ}$ added to vertical axes. Negative results are internally rotated. (For illustration of APA-1, APA-2, and SL, see Fig. 5)

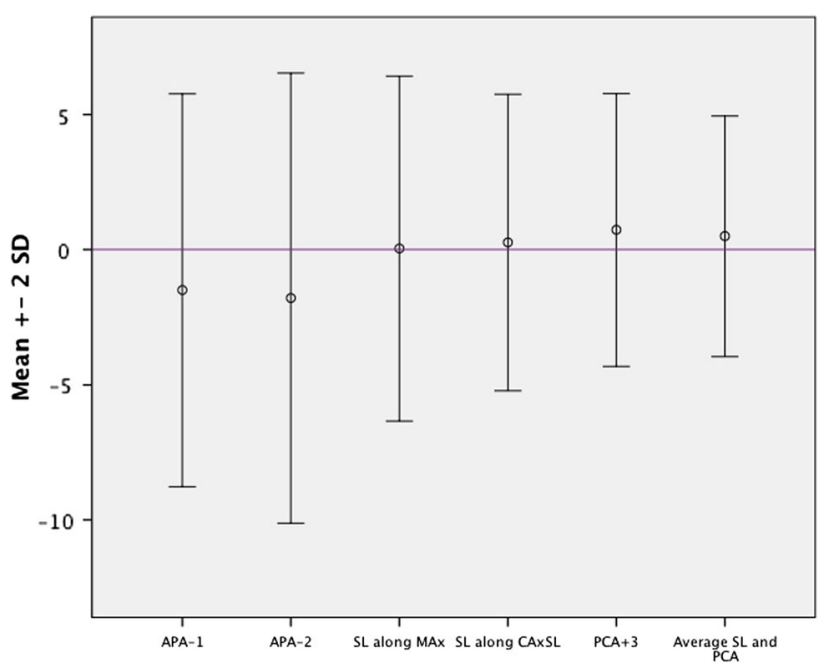

Fig. 8 Mean and standard deviation of each landmark relative to the SEA 
Table 4 Outliers $>3^{\circ}$ from SEA

\begin{tabular}{lll}
\hline & $\begin{array}{l}\text { Outliers }>3^{\circ} \\
\text { from SEA }\end{array}$ & $\begin{array}{l}\text { Percentage } \\
(n=44)(\%)\end{array}$ \\
\hline APA-2 on 3D reconstruction & 19 & 43 \\
PCA $+3^{\circ}$ & 13 & 30 \\
SL along CAxSL & 14 & 32 \\
Average PCA $+3^{\circ}$ and SL & $7^{*}$ & 16 \\
\hline
\end{tabular}

* The average of the PCA $+3^{\circ}$ and SL (PCA-SL) produced significantly less outliers than the $\mathrm{PCA}+3^{\circ}(p=0.03)$ or the SL along CAxSL $(p=0.007)$

Table 5 Coronal axis measurements

\begin{tabular}{lrll}
\hline & Mean & SD & Range \\
\hline CAxSL to MAx & $0.4^{\circ}$ & $3.8^{\circ}$ & $9.4^{\circ}$ to $-7.3^{\circ}$ \\
DCA to MAx & $-2.2^{\circ}$ & $3.1^{\circ}$ & $4.7^{\circ}$ to $-8.1^{\circ}$ \\
CAxSL to DCA & $2.6^{\circ}$ & $4.3^{\circ}$ & $13.7^{\circ}$ to $-4.6^{\circ}$ \\
\hline
\end{tabular}

Negative values are valgus

coronal alignment of the trochlear groove must be considered whenever it is referenced to guide femoral component rotation. Finally, the combination of the SL and the PCA is likely to improve rotational accuracy over either one used individually.

The exclusive use of SL to determine femoral component rotation led to an accurate and reproducible result in a large clinical series. To our knowledge, there are no similar studies assessing the post-operative results achieved using the SL to determine femoral component rotation, thus making comparison difficult.

The only established, valid, and reproducible technique for measuring femoral component rotation is the use of CT scans $[6,8]$. There are few studies (Table 6) that have used $\mathrm{CT}$ scans to assess the post-operative results achieved with the use of any landmarks or techniques [25, 36, 38].

Several studies have compared the use of the measured resection to the gap-balancing techniques using computer navigation or intraoperative measurements to assess the differences [12, 18, 29, 35, 39, 45]. Whilst they have often found large differences between techniques, without comparison with the gold standard of a CT scan of the epicondyles, it is difficult to comment on the relevance of their results.

The second study confirms that when the SL is measured along its own coronal axis (the CAxSL), it is a more accurate landmark for determining the SEA of the femur than the APA. This explains the reasons for the accurate femoral component positioning in the first study despite a large amount of the literature which predicts that referencing the trochlear groove should lead to poor results. The technique of measuring the SL on a 3DCT scan, which is orientated until the SL becomes a straight, vertical line,
Table 6 Comparable research assessing femoral component rotation relative to SEA with post-operative CT scans

\begin{tabular}{llllll}
\hline References & Axis & $N$ & Mean & SD & Range \\
\hline Luyckx [25] & Preoperative CT & 48 & $2.4^{\circ}$ & $2.5^{\circ}$ & $\begin{array}{c}-2.8^{\circ} \\
\text { to } 6.9^{\circ}\end{array}$ \\
& Gap balancing & 48 & $1.7^{\circ}$ & $2.1^{\circ}$ & $\begin{array}{c}-2.5^{\circ} \\
\text { to } 6.5^{\circ}\end{array}$ \\
Stöckl et al. [38] & PCA+3 & 32 & $1.1^{\circ}$ & $2.8^{\circ}$ & $\begin{array}{l}-2^{\circ} \text { to } \\
12^{\circ}\end{array}$ \\
& $\begin{array}{c}\text { APA and } \\
\text { epicondylar }\end{array}$ & 32 & $-0.4^{\circ}$ & $2.4^{\circ}$ & $\begin{array}{c}-7^{\circ} \text { to } \\
4^{\circ}\end{array}$ \\
Sec et al. [36] & $\begin{array}{c}\text { Mechanical axis } \\
\text { derived }\end{array}$ & 120 & $1.6^{\circ}$ & $2.2^{\circ}$ & $\begin{array}{c}-4.8^{\circ} \\
\text { to } 7.9^{\circ}\end{array}$ \\
& & & & &
\end{tabular}

reflects the clinical use of the SL during surgery (Figs. 1, 2, $3)$. The trochlear groove is a three-dimensional arc which has a rotational component and a coronal orientation. In comparison, referencing the groove with two points such as the APA [3, 43] is likely to lead to additional variability due to both anatomical and geometrical reasons. This is shown in Fig. 9 which show the APA marked on the same femur viewed from two different coronal viewpoints. A parallax error causes the angle between the APA and the fixed epicondylar axis to vary between the two viewpoints. This error is extremely difficult to detect intraoperatively and has not been accounted for in previous studies measuring the APA.

There are two anatomical interpretations that have been used in studies of the APA. Nagamine et al. [27] determined the APA on a single 2D axial CT slice (Fig. 4). They isolated the slice that showed the epicondyles most prominently and then measured the low point in the groove anteriorly to the high point in the notch posteriorly. Anatomically, the posterior point localised with this technique is deep within the intercondylar notch and is $1-2 \mathrm{~cm}$ posterior to the articular surface of the trochlear groove (Fig. 5, APA-1). This posterior point is not readily accessible during surgery, which makes this measurement less clinically relevant. We recreated this technique and obtained a mean of $-1.5^{\circ}$ and a SD of $3.6^{\circ}$. These results are very similar to those obtained by Nagamine et al. [27] who reported a mean of $-1.4^{\circ}$ and a SD of $3.3^{\circ}$.

Most other studies have used a posterior point on the articular surface in the floor of the trochlear groove immediately anterior to the intercondylar notch. This is the same starting point as the SL. However, the anterior point used to measure the APA is the deepest point of the trochlear groove anteriorly (Fig. 5, APA-2). This point references the most proximal section of the trochlear groove that is more variable in normal knees and more prone to bone erosion and osteophyte formation in osteoarthritic knees. Our results confirm the inconsistency of the 

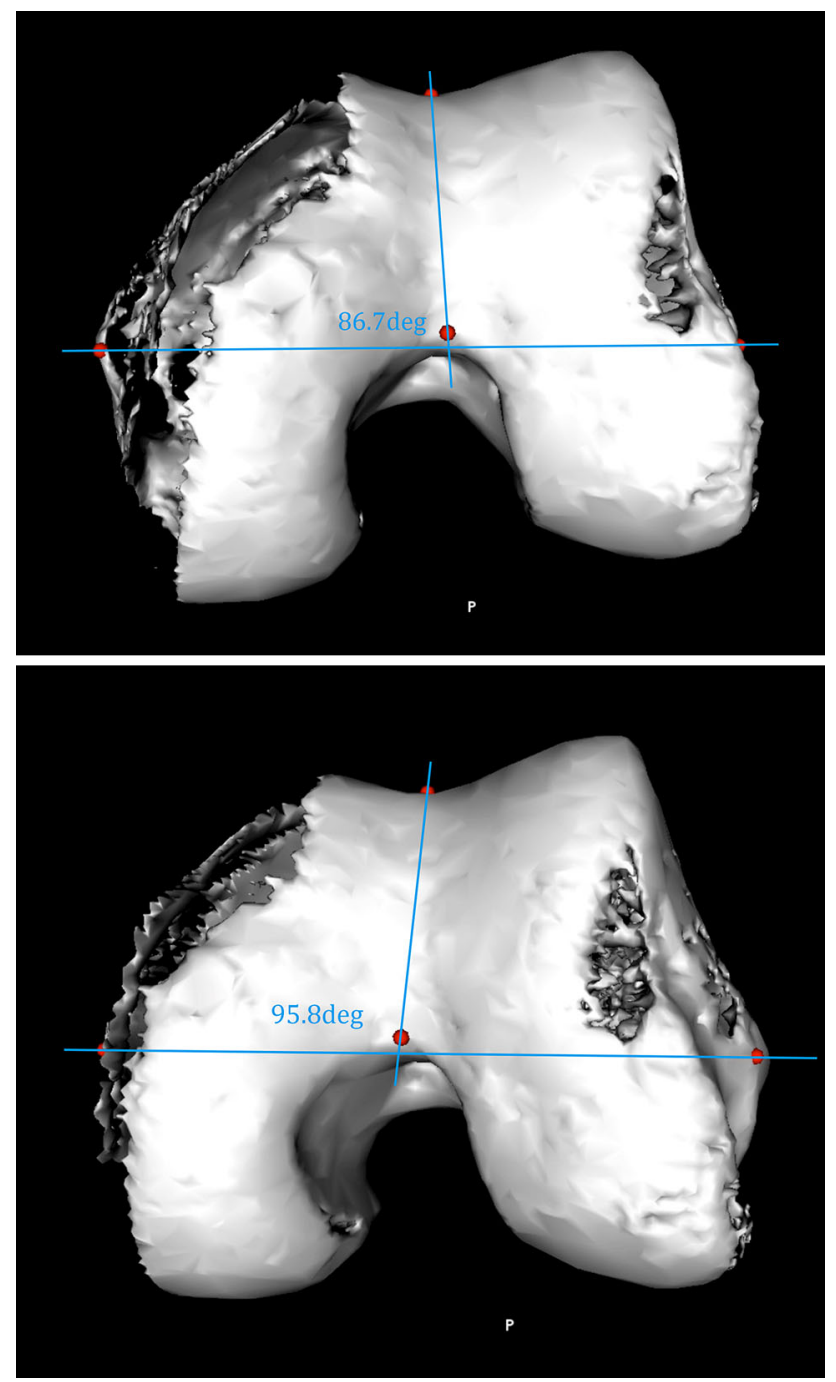

Fig. 9 Due to parallax error the rotational angle of the APA relative to a fixed landmark such as the epicondylar axis changes as the coronal viewpoint changes

anterior point of the APA. We measured the APA on 3D reconstructions, aligned to the MAx, from the posterior point in the trochlear groove to the deepest point in the trochlear groove anteriorly. This produced a mean of $-1.8^{\circ}$ and a SD of $4.2^{\circ}$ compared with the SL measured along the same axis which produced a mean of $0.0^{\circ}\left(\mathrm{SD} 3.2^{\circ}\right)$. It was frequently noted that the most proximal section deviated noticeably from the rest of the groove and that it was often affected by osteophytes and bone erosion.

The inconsistency of the anterior point in the APA is supported by Victor et al. [41] who found considerably poorer intraobserver and interobserver reliability for detecting the deepest point of the trochlear groove anteriorly than for detecting the knee centre in the groove distally. Cerveri et al. [9] also reported that the interobserver reliability for detecting the point in the anterior trochlear groove was significantly worse than for the distal point.
Cerveri et al. [9] also demonstrated improved repeatability of a computer-derived technique for marking multiple points along the trochlear groove in comparison with the APA as measured by surgeons marking only two points. Piriou et al. [32] recently obtained consistent results by referencing several points along the trochlear groove using computer navigation instead of relying on a single anterior point.

The key anatomical differences between the SL and the APA are highlighted by research that measured the APA after the distal femoral resection [37, 44]. By removing the distal femur, the majority of the vertical component of the trochlear groove, that is essential in producing the SL, is removed.

The geometrical advantage of the SL occurs due to the use of multiple points along the floor of the trochlear groove. This allows the orientation of the trochlear groove to its own coronal axis, which is impossible to achieve with the APA. The variations between the APA and the epicondylar axes caused by the alteration in the coronal viewpoint can be illustrated by comparing oblique views of the reconstructions (Figs. 1, 2,9). This concept may explain the findings of Iranpour et al. [20] when they noted that the coronal orientation that leads to the least medial and lateral variability of the multiple points measured along the trochlear groove was the orientation along the groove itself.

Crucial to the concept of isolating the rotational component of the trochlear groove from the coronal component is the variability in the coronal alignment of the SL. This was discussed by Iranpour et al. [20] who used a similar technique for identifying multiple points in the sulcus. They compared the coronal alignment of the SL to the coronal alignment of a DCA derived from sphere matching to the condyles. They found a mean of $0^{\circ}$ but a large $\mathrm{SD}$ of $5^{\circ}$. In the current study, we compared the CAxSL to the MAx and found a mean of $0.4^{\circ}$ (SD $3.8^{\circ}$, range from $-7.3^{\circ}$ to $9.4^{\circ}$ ). We also compared the CAxSL to a line perpendicular to the DCA, mean $2.6^{\circ}$ (SD $4.3^{\circ}$, range from $-13.7^{\circ}$ to $4.6^{\circ}$ ). These very wide ranges are consistent with the work of Iranpour et al. [20]. The significance of this variation is that it effects both the measurement of the APA or SL in anatomical studies and also the intraoperative use of the SL.

When using the SL during surgery, the line is usually translated into a horizontal line on the distal condylar surface, as per the technique described previously (Fig. 3) [39]. Since identifying the variability in the CAxSL relative to the DCA, it has become apparent that there is a further geometrical flaw in this technique. This error is separate to the geometrical error caused when observing the SL from an orientation other than along the CAxSL that we have described above. This translating error will occur during surgery even though we are orientated along the coronal alignment of the SL. Whenever transferring the rotational line from one coronal plane (the CAxSL) on to 
another (the DCA), there is a risk of changing the rotational angle. This will occur whenever there is also a deviation in the sagittal plane.

In practice, this occurs when using a device such as the T-piece to transfer the vertical SL into a horizontal line on the surface of the condyles [39]. If there is any flexion or extension in the sagittal plane of the T-piece, relative to the planned distal femoral cut, in combination with any difference between the coronal alignment of the SL and the DCA, then a rotational error will occur. This error can be calculated from Euler's rotational theorem using the formula, $\tan \theta_{3}=\sin \theta_{1} \sin \theta_{2} / \cos \theta_{1}$ where $\theta_{1}=$ coronal plane variation (CAxSL-DCA), $\theta_{2}=$ sagittal plane variation of T-piece to planned distal femoral cut, and $\theta_{3}=$ resultant rotational variation in axial plane. ${ }^{1}$ In practice, the error produced with the intraoperative use of a T-piece is likely to be several degrees (Fig. 10). It becomes more likely in patients with a large divergence between the DCA and the CAxSL (Fig. 11).

A trochlear alignment guide (TAG) has been developed that corrects for the geometrical errors inherent in the use of the SL during surgery and transfers that angle onto the cut distal surface of the femur. This allows direct comparison of the geometrically accurate SL with the PCA. From the results of the 3D CT study, we anticipate that the averaging of these axes will decrease the likelihood of femoral component malrotation. Similar results have recently been shown by combining the APA and PCA using PSI [31]. We predict that even greater accuracy may be achieved by using a geometrically correct SL instead of the APA. The use of the TAG allows the SL and PCA to be combined using conventional instruments rather than only with PSI.

These concepts have implications for current techniques of measuring the APA or SL on CT scans and MRI scans and for their use in computer navigation systems. The reference of the APA during landmark identification associated with computer navigation could be improved by compensating for the variation between the coronal alignment of the groove and the MAx. With the increasing popularity of patient-specific instruments based on preoperative CT and MRI scans, we believe that this geometrical concept must be included in the measurement algorithms if the trochlear groove is being used to assist in rotational alignment.

These studies have a number of limitations. In the first study, our use of two-dimensional axial CT slices to measure the rotational alignment of the femoral component

\footnotetext{
${ }^{1}$ Euler's theorem and its proof are contained in paragraphs 24-26 of the appendix (Additamentum. pp. 201-203) of L. Eulero (Leonhard Euler), Formulae generales pro translatione quacunque corporum rigidorum (General formulas for the translation of arbitrary rigid bodies), presented to the St. Petersburg Academy on October 9, 1775.
}
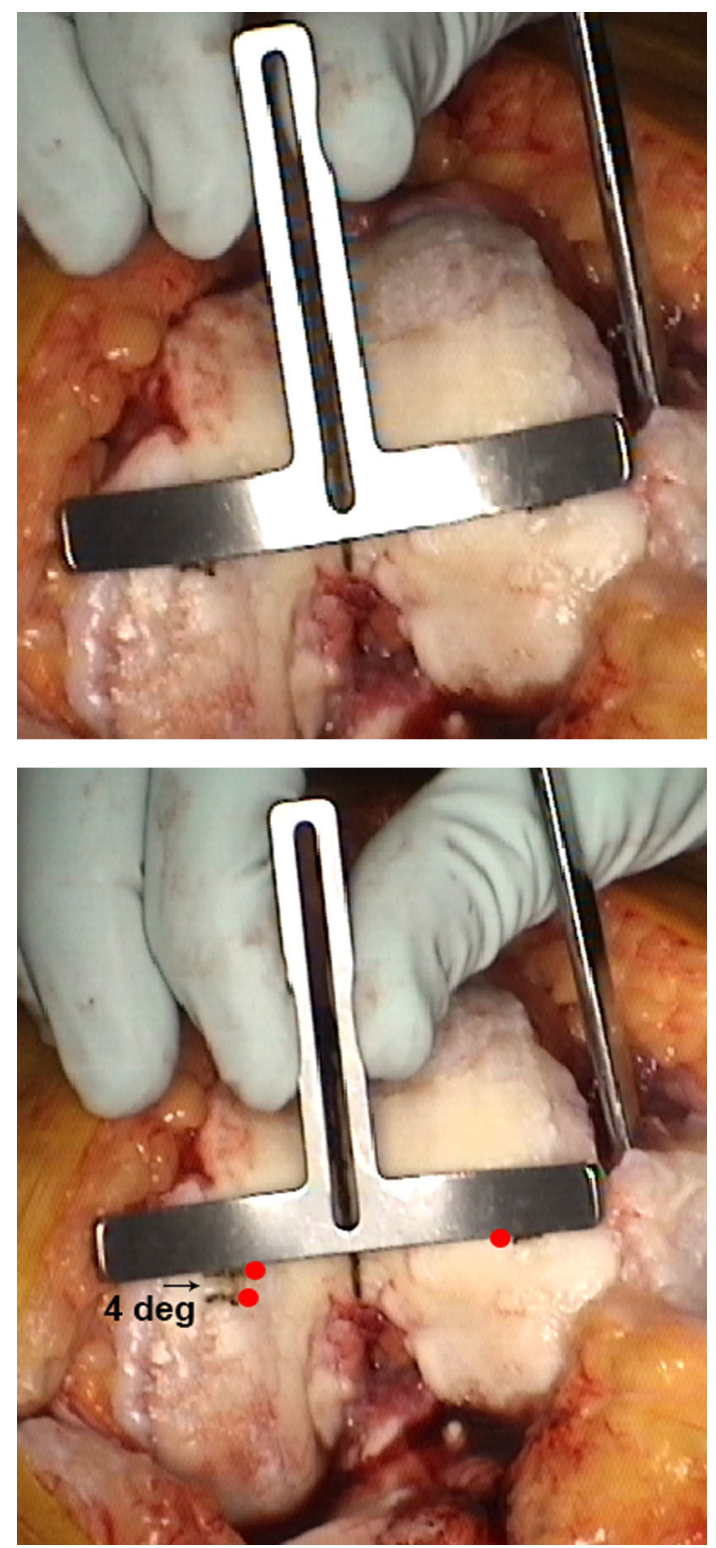

Fig. 10 The rotational angle of the horizontal limb of the T-piece will change with flexion or extension of the T-piece even though the vertical limb stays aligned with the SL

is likely to lead to a measurement error that could be reduced with $3 \mathrm{D}$ reconstructions. In addition, due to the presence of metal artefact, 28 scans were excluded. There is no comparison group in the first study, and the surgeon is very experienced in this technique; therefore, the findings may not extend to other surgeons. However, the study has a number of strengths. To our knowledge, this is the largest study to use post-operative CT scans to assess femoral component rotation and it is also the only study assessing the radiological outcomes from the use of the SL, making the findings novel.

The 3D CT study relies on meticulous positioning of the points along the trochlear groove, which are then 


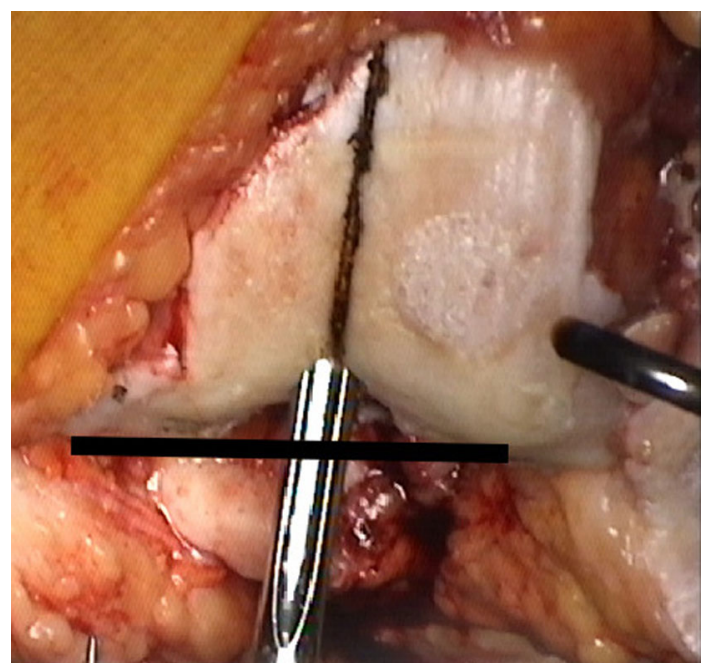

Fig. 11 In this case the CAxSL parallels the intramedullary rod (anatomical axis of the femur) and is widely divergent from the DCA (black line)

checked on each axis view and on the reconstructions. The use of CT scans disregards the contribution of articular cartilage to the shape of the trochlear groove. However, with MRI scans, it is very difficult to accurately compare the coronal alignment of the trochlear groove to the MAx of the femur.

\section{Conclusion}

These findings demonstrate the importance of understanding the three-dimensional nature of the trochlear groove. When this concept is appreciated, the groove is a reliable landmark for determining femoral component rotation. Additional accuracy can be obtained by combining the PCA with a geometrically correct SL.

\section{References}

1. Aglietti P, Buzzi R, Gaudenzi A (1988) Patellofemoral functional results and complications with the posterior stabilized total condylar knee prosthesis. J Arthroplasty 3(1):17-25

2. Anouchi YS, Whiteside LA, Kaiser AD, Milliano MT (1993) The effects of axial rotational alignment of the femoral component on knee stability and patellar tracking in total knee arthroplasty demonstrated on autopsy specimens. Clin Orthop Relat Res 287:170-177

3. Arima J, Whiteside LA, McCarthy DS, White SE (1995) Femoral rotational alignment, based on the anteroposterior axis, in total knee arthroplasty in a valgus knee. A technical note. J Bone Joint Surg Am 77(9): 1331-1334

4. Asano T, Akagi M, Nakamura T (2005) The functional flexionextension axis of the knee corresponds to the surgical epicondylar axis: in vivo analysis using a biplanar image-matching technique. J Arthroplasty 20(8):1060-1067
5. Barrack RL, Schrader T, Bertot AJ, Wolfe MW, Myers L (2001) Component rotation and anterior knee pain after total knee arthroplasty. Clin Orthop Relat Res 392:46-55

6. Berger RA, Crossett LS (1998) Determining the rotation of the femoral and tibial components in total knee arthroplasty: a computer tomography technique. Oper Tech Orthop 8(3):128-133

7. Berger RA, Crossett LS, Jacobs JJ, Rubash HE (1998) Malrotation causing patellofemoral complications after total knee arthroplasty. Clin Orthop Relat Res 356:144-153

8. Berger RA, Rubash HE, Seel MJ, Thompson WH, Crossett LS (1993) Determining the rotational alignment of the femoral component in total knee arthroplasty using the epicondylar axis. Clin Orthop Relat Res 286:40-47

9. Cerveri P, Marchente M, Manzotti A, Confalonieri N (2011) Determination of the Whiteside line on femur surface models by fitting high-order polynomial functions to cross-section profiles of the intercondylar fossa. Comput Aided Surg 16(2):71-85

10. Chauhan SK, Clark GW, Scott RG, Lloyd S, Sikorski JM, Breidahl W (2004) The Perth CT Protocol for total knee replacement. J Bone Joint Surg Br 86-B(SUPP IV):442-443

11. Churchill DL, Incavo SJ, Johnson CC, Beynnon BD (1998) The transepicondylar axis approximates the optimal flexion axis of the knee. Clin Orthop Relat Res 356:111-118

12. Dennis DA, Komistek RD, Kim RH, Sharma A (2010) Gap balancing versus measured resection technique for total knee arthroplasty. Clin Orthop Relat Res 468(1):102-107

13. Dorr LD, Boiardo RA (1986) Technical considerations in total knee arthroplasty. Clin Orthop Relat Res 205:5-11

14. Fehring TK (2000) Rotational malalignment of the femoral component in total knee arthroplasty. Clin Orthop Relat Res 380:72-79

15. Figgie HE 3rd, Goldberg VM, Figgie MP, Inglis AE, Kelly M, Sobel M (1989) The effect of alignment of the implant on fractures of the patella after condylar total knee arthroplasty. J Bone Joint Surg Am 71(7):1031-1039

16. Griffin FM, Insall JN, Scuderi GR (1998) The posterior condylar angle in osteoarthritic knees. J Arthroplasty 13(7):812-815

17. Griffin FM, Math K, Scuderi GR, Insall JN, Poilvache PL (2000) Anatomy of the epicondyles of the distal femur: MRI analysis of normal knees. J Arthroplasty 15(3):354-359

18. Heesterbeek PJC, Jacobs WCH, Wymenga AB (2009) Effects of the balanced gap technique on femoral component rotation in TKA. Clin Orthop Relat Res 467(4):1015-1022

19. Hungerford DS, Kenna RV (1983) Preliminary experience with a total knee prosthesis with porous coating used without cement. Clin Orthop 176:95-107

20. Iranpour F, Merican A, Dandachli W, Amis A, Cobb J (2010) The geometry of the trochlear groove. Clin Orthop Relat Res 468(3):782-788

21. Jenny J-Y, Boeri C (2004) Low reproducibility of the intraoperative measurement of the transepicondylar axis during total knee replacement. Acta Orthop Scand 75(1):74-77

22. Jerosch J, Peuker E, Philipps B, Filler T (2002) Interindividual reproducibility in perioperative rotational alignment of femoral components in knee prosthetic surgery using the transepicondylar axis. Knee Surg Sports Traumatol Arthrosc 10(3):194-197

23. Kinzel V, Ledger M, Shakespeare D (2005) Can the epicondylar axis be defined accurately in total knee arthroplasty? Knee 12(4):293-296

24. Laskin RS (1995) Flexion space configuration in total knee arthroplasty. J Arthroplasty 10(5):657-660

25. Luyckx T, Peeters T, Vandenneucker H, Victor J, Bellemans J (2012) Is adapted measured resection superior to gap-balancing in determining femoral component rotation in total knee replacement? J Bone Joint Surg Br 94(9):1271-1276 
26. Moon Y-W, Ha C-W, Do K-H, Kim C-Y, Han J-H, Na S-E, Lee C-H, Kim J-G, Park Y-S (2012) Comparison of robot-assisted and conventional total knee arthroplasty: a controlled cadaver study using multiparameter quantitative three-dimensional CT assessment of alignment. Comput Aided Surg 17(2):86-95

27. Nagamine R, Miura H, Inoue Y, Urabe K, Matsuda S, Okamoto Y, Nishizawa M, Iwamoto Y (1998) Reliability of the anteroposterior axis and the posterior condylar axis for determining rotational alignment of the femoral component in total knee arthroplasty. J Orthop Sci 3(4):194-198

28. Olcott CW, Scott RD (1999) The Ranawat Award. Femoral component rotation during total knee arthroplasty. Clin Orthop Relat Res 367:39-42

29. Olcott CW, Scott RD (2000) A comparison of 4 intraoperative methods to determine femoral component rotation during total knee arthroplasty. J Arthroplasty 15(1):22-26

30. Oussedik S, Scholes C, Ferguson D, Roe J, Parker D (2012) Is femoral component rotation in a TKA reliably guided by the functional flexion axis? Clin Orthop Relat Res 470(11): 3227-3232

31. Paternostre F, Schwab P-E, Thienpont E (2014) The combined Whiteside's and posterior condylar line as a reliable reference to describe axial distal femoral anatomy in patient-specific instrument planning. Knee Surg Sports Traumatol Arthrosc. doi:10. 1007/s00167-014-2836-5

32. Piriou P, Peronne E, Ouanezar H (2013) Rotational alignment of the femoral component using trochlear navigation during total knee arthroplasty: a dual-center study of 145 cases. J Arthroplasty 28(7):1107-1111

33. Poilvache PL, Insall JN, Scuderi GR, Font-Rodriguez DE (1996) Rotational landmarks and sizing of the distal femur in total knee arthroplasty. Clin Orthop Relat Res 331:35-46

34. Ranawat CS (1986) The patellofemoral joint in total condylar knee arthroplasty. Pros and cons based on five- to ten-year follow-up observations. Clin Orthop Relat Res 205:93-99

35. Schnurr C, Nessler J, Konig DP (2009) Is referencing the posterior condyles sufficient to achieve a rectangular flexion gap in total knee arthroplasty? Int Orthop 33(6):1561-1565
36. Seo J-G, Moon Y-W, Lim J-S, Park S-J, Kim S-M (2012) Mechanical axis-derived femoral component rotation in extramedullary total knee arthroplasty: a comparison between femoral transverse axis and transepicondylar axis. Knee Surg Sports Traumatol Arthrosc 20(3):538-545

37. Siston RA, Patel JJ, Goodman SB, Delp SL, Giori NJ (2005) The variability of femoral rotational alignment in total knee arthroplasty. J Bone Joint Surg Am 87(10):2276-2280

38. Stockl B, Nogler M, Rosiek R, Fischer M, Krismer M, Kessler O (2004) Navigation improves accuracy of rotational alignment in total knee arthroplasty. Clin Orthop Relat Res 426:180-186

39. Talbot S, Bartlett $\mathbf{J}$ (2008) The anterior surface of the femur as a new landmark for femoral component rotation in total knee arthroplasty. Knee Surg Sports Traumatol Arthrosc 16(3):258-262

40. Victor J (2009) Rotational alignment of the distal femur: a literature review. Orthop Traumatol Surg Res 95(5):365-372

41. Victor J, Van Doninck D, Labey L, Innocenti B, Parizel PM, Bellemans J (2009) How precise can bony landmarks be determined on a CT scan of the knee? Knee 16(5):358-365

42. Victor J, Van Doninck D, Labey L, Van Glabbeek F, Parizel P, Bellemans J (2009) A common reference frame for describing rotation of the distal femur: a ct-based kinematic study using cadavers. J Bone Joint Surg Br 91(5):683-690

43. Whiteside LA, Arima J (1995) The anteroposterior axis for femoral rotational alignment in valgus total knee arthroplasty. Clin Orthop Relat Res 321:168-172

44. Wraighte PJ, Sikand M, Livesley PJ (2011) Intra- and interobserver variation during femoral jig rotational alignment in knee arthroplasty. Arch Orthop Trauma Surg 131(9):1283-1286

45. Yau WP, Chiu KY, Tang WM (2007) How precise is the determination of rotational alignment of the femoral prosthesis in total knee arthroplasty: an in vivo study. J Arthroplasty 22(7):1042-1048

46. Yoshioka Y, Siu D, Cooke TD (1987) The anatomy and functional axes of the femur. J Bone Joint Surg Am 69(6):873-880 